\title{
Effect of interlayer exchange coupling on magnetization reversal process in ECC media with high coercivity
}

\author{
A. Oyama ${ }^{1}$, T. Komine ${ }^{1}$, and R. Sugita ${ }^{1}$ \\ ${ }^{1}$ Ibaraki Univ., 4-12-1 Nakanarusawa-cho, Hitachi, Ibaraki 316-8511, Japan
}

\begin{abstract}
Effect of interlayer exchange coupling on time-evolutional magnetization reversal process in ECC media with high coercivity was investigated by utilizing micromagnetic simulation. In the case of intermediate value of the interlayer exchange coupling, during the application of printing field, magnetization in the soft layer initially reverses and magnetization in the hard layer follows that of the soft layer. Furthermore, after removal of printing field, magnetization in the soft layer is reversed again according with that in the hard layer.
\end{abstract}

\section{Introduction}

The exchange-coupled composite (ECC) media [1] are still a strong candidate for achieving ultra-high recording density in hard disks with high coercivity [2]. Berger et al. investigated the effect of interlayer exchange coupling on the recording performance in the ECC media by experiments [3]. Moreover, Inaba et al. reported that the interlayer exchange coupling is closely related to magnetization reversal process in the ECC media, and that the magnetization reversal process is divided into three modes, which are spin-flop, incoherent, coherent rotation modes [4]. However, although the magnetization reversed process in the ECC media is one of important issues in order to achieve further ultra-high recording density, the time-evolutional magnetization process in the ECC media with high coercivity has not been sufficiently discussed yet. In this study, effect of interlayer exchange coupling on time-evolutional magnetization reversal process in the ECC media with high coercivity was investigated by utilizing micromagnetic simulation.

\section{Calculation method}

In order to investigate magnetization reversal process in the ECC media, magnetic printing [5] was used as recording technique in the simulation. In magnetic printing, first, the recording layer is magnetized by applying the initial magnetic field along the perpendicular direction of recording layer. Then the master medium with a patterned magnetic layer corresponding to servo signals is in contact with the recoding layer, and after that, the printing field is applied along the opposite direction to the initial magnetic field. Finally, the servo patterns are printed into the recording layer. Fig. 1 shows the simulation model of magnetic printing used in the study. The master pattern has the track width of $30 \mathrm{~nm}$ and the bit length of $30 \mathrm{~nm}$. In this study, as shown in Fig. 1, the simulation model of the recording layer consists of the soft and hard layers. The recording layer was divided into $5 \times 5 \times 5 \mathrm{~nm}^{3}$ cubic cells for the simulation. Exchange lengths of the soft and hard layers are comparable to the cell size in this simulation. Table 1 shows parameters of the ECC medium. The ECC medium with coercivity of about $10 \mathrm{kOe}$ was assumed. The interlayer exchange coupling constant $A_{\text {interlayer was }}$ varied from $1.0 \times 10^{-7}$ to $10.0 \times 10^{-7} \mathrm{erg} / \mathrm{cm}$. The timeevolutional magnetization reversal process in the soft and

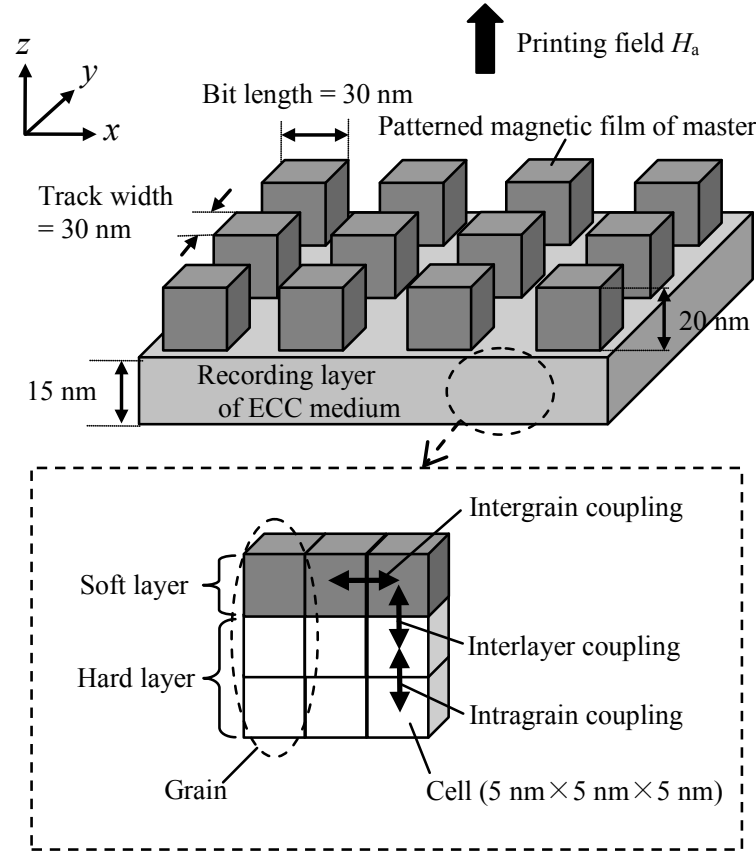

Fig. 1. Simulation model of magnetic printing.

This is an Open Access article distributed under the terms of the Creative Commons Attribution License 2.0, which permits unrestricted use, distribution, and reproduction in any medium, provided the original work is properly cited. 
Table 1. Parameters of ECC medium used in the study.

\begin{tabular}{|c|c|c|c|}
\hline & & Soft layer & Hard layer \\
\hline \multicolumn{2}{|l|}{ Thickness (nm) } & 5 & 10 \\
\hline \multicolumn{2}{|c|}{ Saturation magnetization $M_{\mathrm{s}}\left(\mathrm{emu} / \mathrm{cm}^{3}\right)$} & 600 & 600 \\
\hline \multicolumn{2}{|c|}{ Anisotropy field $H_{\mathrm{k}}(\mathrm{kOe})$} & 8 & 20 \\
\hline \multicolumn{2}{|c|}{ Dispersion of anisotropy field $\Delta H_{\mathrm{k}}(\mathrm{kOe})$} & 0.8 & 2.0 \\
\hline \multicolumn{2}{|c|}{ c-axis distribution $\Delta \theta_{50}$ (deg.) } & 10 & 10 \\
\hline \multirow{3}{*}{ Exchange coupling constant $\left(\times 10^{-7} \mathrm{erg} / \mathrm{cm}\right)$} & $A_{\text {intergrain }}$ & 1.0 & 0.5 \\
\hline & $A_{\text {intragrain }}$ & - & 10.0 \\
\hline & $A_{\text {interlayer }}$ & \multicolumn{2}{|c|}{$1.0-10.0$} \\
\hline
\end{tabular}

hard layers was analyzed during application of printing field $H_{\mathrm{a}}$ and after removal of $H_{\mathrm{a}}$. Printing performance PP was evaluated from the calculated magnetization distribution in the soft and hard layers. The PP in each layer was estimated by using the following definition [6]:

$$
\mathrm{PP}(\%)=\frac{\sum M_{z}^{\text {ideal }} M_{z}^{\text {cal }}}{\sum M_{z}^{\text {ideal }} M_{z}^{\text {ideal }}} \times 100,
$$

where $M_{\mathrm{z}}^{\text {ideal }}$ and $M_{\mathrm{z}}^{\mathrm{cal}}$ are $z$-component of ideally printed magnetization and $z$-component of calculated magnetization, respectively. The PP means whether the calculated magnetization is close to the ideal magnetization. When the printed magnetization is ideal, the value of PP is $100 \%$.

\section{Results and discussion}

Fig. 2 shows the hysteresis loops of the ECC media with various $A_{\text {interlayer }}$ Magnetization of the soft and hard layers is simultaneously reversed due to the strong interlayer exchange coupling between two layers when $A_{\text {interlayer }}$ is $10.0 \times 10^{-7} \mathrm{erg} / \mathrm{cm}$. Such a magnetization reversal process is hereafter denoted as coherent rotation [4]. Magnetization reversal in the soft layer occurs faster compared to the hard layer when $A_{\text {interlayer }}$ is $5.0 \times 10^{-7}$ $\mathrm{erg} / \mathrm{cm}$. In this case the magnetization distribution in the soft layer is finally consistent with that of the hard layer, and the coercivity is less than that in the coherent rotation mode. Such a magnetization reversal process is denoted as incoherent rotation. Meanwhile magnetization of each layer is independently reversed when $A_{\text {interlayer }}$ is $1.0 \times 10^{-7}$ $\mathrm{erg} / \mathrm{cm}$. Such a magnetization reversal process is denoted as spin-flop rotation. In any case, the squareness ratio is 1 in the simulation.

Fig. 3 shows magnetization change with time for $A_{\text {interlayer }}$ of $1.0 \times 10^{-7} \mathrm{erg} / \mathrm{cm}$ and $H_{\mathrm{a}}$ of $1 \mathrm{kOe}$. During the application of $H_{\mathrm{a}}$, magnetization of the soft layer is reversed as shown in Figs. 3(a) and 3(b). Whereas, the magnetization of the hard layer does not change at all. Namely, magnetization of each layer is independently reversed. Magnetization of each layer has not changed after removal of $H_{\mathrm{a}}$ (Fig. 3(c)). Fig. 4 shows the timeevolutional PP with lapse of time in the soft and hard layers when $A_{\text {interlayer is } 1.0 \times 10^{-7}} \mathrm{erg} / \mathrm{cm}$. The magnetization of the soft layer is printed in accordance with the pattern of master, while the hard layer is hardly printed.

Fig. 5 shows magnetization change with time for $A_{\text {interlayer }}$ of $5.0 \times 10^{-7} \mathrm{erg} / \mathrm{cm}$ and $H_{\mathrm{a}}$ of $4.5 \mathrm{kOe}$. When $H_{\mathrm{a}}$ is applied, magnetization reversal in the soft layer occurs (Fig. 5(a)), and then magnetization reversal in the hard layer follows that of the soft layer (Fig. 5(b)). Finally, magnetization of the soft layer is affected by that of the hard layer and coincides with it after removal of $H_{\mathrm{a}}$ (Fig. 5(c)). Fig. 6 shows the time-evolutional PP with lapse of time in the soft and hard layers when $A_{\text {interlayer }}$ is $5.0 \times 10^{-7}$

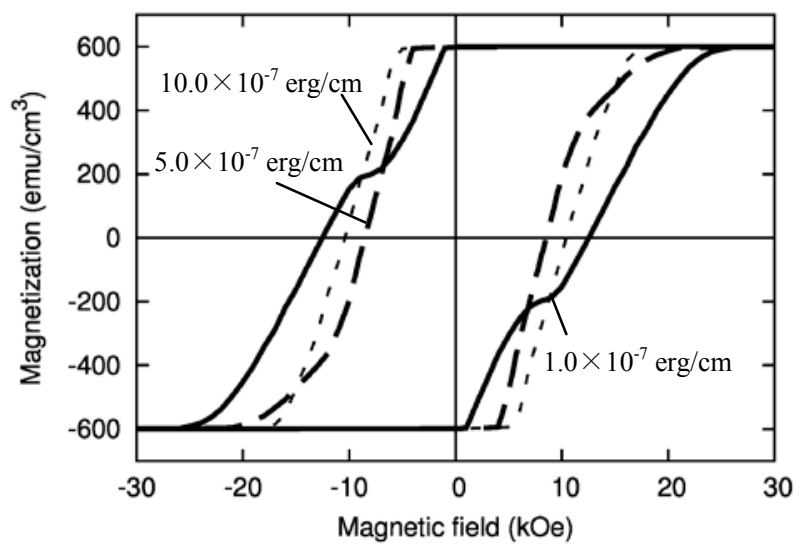

Fig. 2. Perpendicular hysteresis loops with various $A_{\text {interlayer. }}$

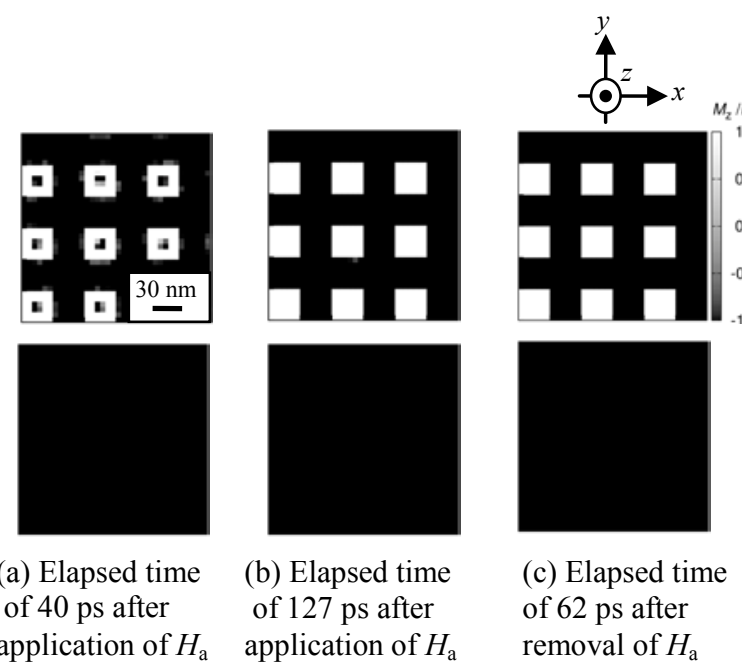

Fig. 3. Magnetization change with a lapse of time. Each top figure shows magnetization distribution in the soft layer, and each bottom figure shows that of the hard layer $\left(A_{\text {interlayer }}=\right.$ $\left.1.0 \times 10^{-7} \mathrm{erg} / \mathrm{cm}\right)$. 


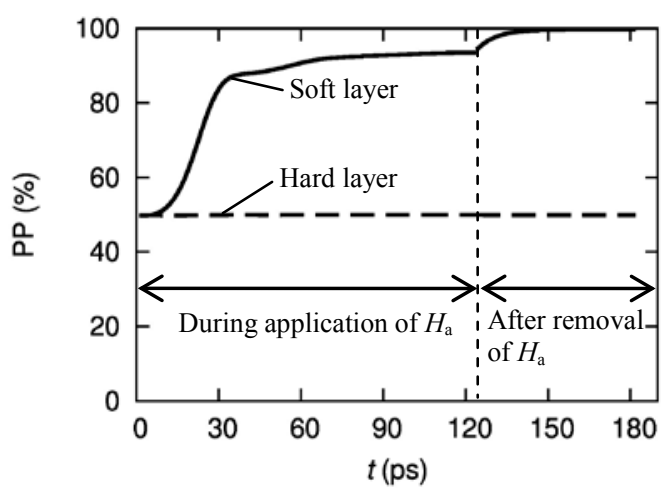

Fig. 4. Printing performance with lapse of time in the soft and hard layers $\left(A_{\text {interlayer }}=1.0 \times 10^{-7} \mathrm{erg} / \mathrm{cm}\right)$.

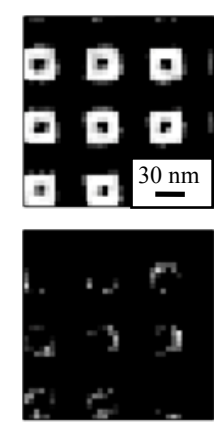

(a) Elapsed time of 30 ps after application of $H$
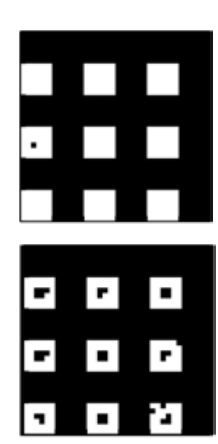

(b) Elapsed time of 105 ps after application of $H_{\mathrm{a}}$
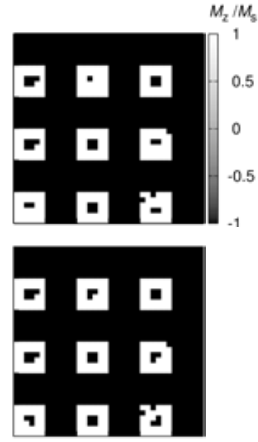

(c) Elapsed time of $130 \mathrm{ps}$ after removal of $H_{\mathrm{a}}$
Fig. 5. Magnetization change with a lapse of time. Each top figure shows magnetization distribution in the soft layer, and each bottom figure shows that of the hard layer $\left(A_{\text {interlayer }}=\right.$ $\left.5.0 \times 10^{-7} \mathrm{erg} / \mathrm{cm}\right)$.

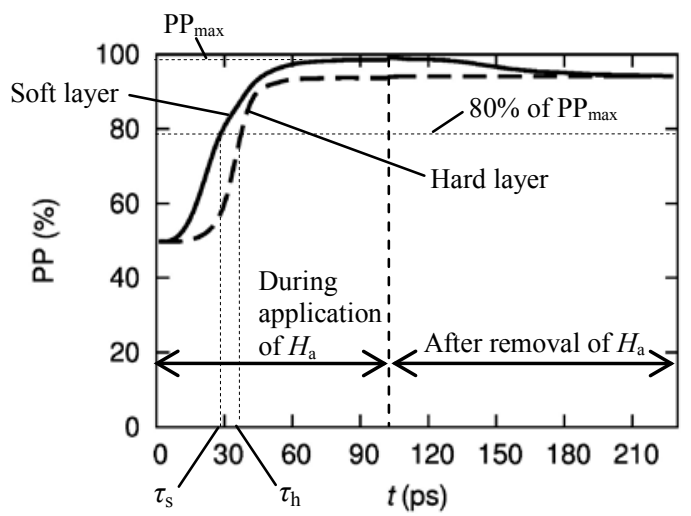

Fig. 6. Printing performance with lapse of time in the soft and hard layers $\left(A_{\text {interlayer }}=5.0 \times 10^{-7} \mathrm{erg} / \mathrm{cm}\right)$.

erg/cm. During the application of $H_{\mathrm{a}}$, magnetization of the soft layer is printed in accordance with the pattern of master. Magnetization of the hard layer follows slightly behind that of the soft layer. After removal of $H_{\mathrm{a}}$, magnetization of the soft layer coincides with that of thehard layer. This issue will be discussed in detail later.

Fig. 7 shows magnetization change with time for

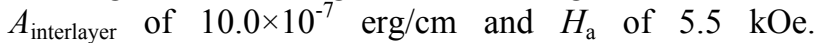
Magnetization of the soft and hard layers is almost simultaneously reversed as shown in Figs. 7(a) and 7(b) when $H_{\mathrm{a}}$ is applied. Magnetization of each layer is the same after removal of $H_{\mathrm{a}}$ (Fig. 7(c)). Fig. 8 shows the

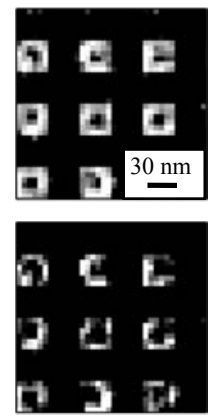

(a) Elapsed time of $30 \mathrm{ps}$ after application of $H_{\mathrm{a}}$
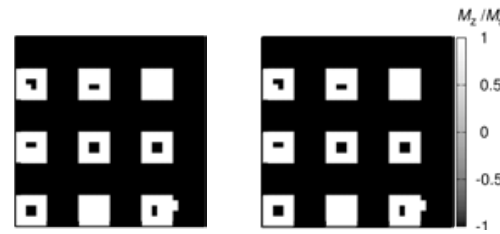

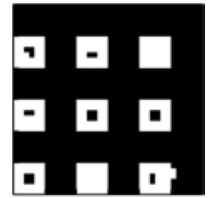

(b) Elapsed time of 91 ps after application of $H_{\mathrm{a}}$

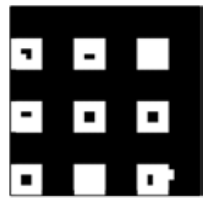

(c) Elapsed time of $30 \mathrm{ps}$ after removal of $H_{\mathrm{a}}$
Fig. 7. Magnetization change with a lapse of time. Each top figure shows magnetization distribution in the soft layer, and each bottom figure shows that of the hard layer $\left(A_{\text {interlayer }}=\right.$ $\left.10.0 \times 10^{-7} \mathrm{erg} / \mathrm{cm}\right)$.

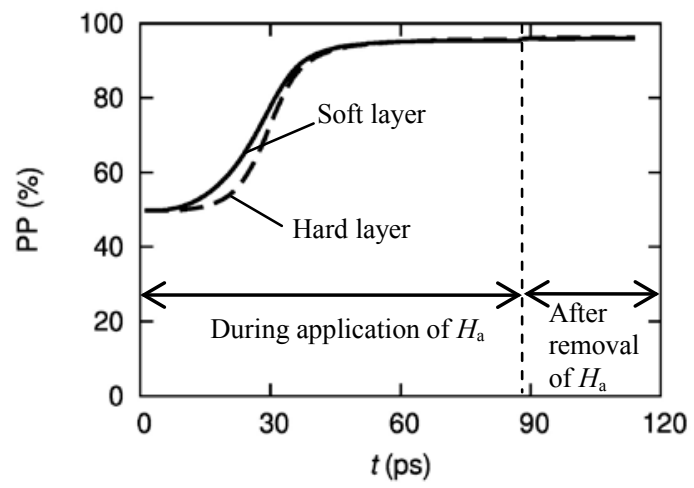

Fig. 8. Printing performance with lapse of time in the soft and hard layers $\left(A_{\text {interlayer }}=10.0 \times 10^{-7} \mathrm{erg} / \mathrm{cm}\right)$.

time-evolutional PP with lapse of time in the soft and hard layers when $A_{\text {interlayer }}$ is $10.0 \times 10^{-7} \mathrm{erg} / \mathrm{cm}$. Both curves for the soft and hard layers almost coincide.

In order to systematically discuss the magnetization reversal in the soft and hard layers, herein, delay parameter $\mathrm{D}$ is defined as $\mathrm{D}=\left(\tau_{\mathrm{h}}-\tau_{\mathrm{s}}\right) / \tau_{\mathrm{s}}$. Here $\tau_{\mathrm{h}}$ and $\tau_{\mathrm{s}}$ are defined as the time that the PP of the hard layer and the PP of the soft layer reach $80 \%$ of maximum value of the $\mathrm{PP}\left(\mathrm{PP}_{\max }\right)$ during application of $H_{\mathrm{a}}$ in the soft layer, respectively, as shown in Fig. 6. Fig. 9 shows the dependence of the $\mathrm{D}$ on $A_{\text {interlayer. Note that }}$. N is infinite based on the definition when the magnetization in the hard layer is not reversed. The $\mathrm{D}$ is infinite when $A_{\text {interlayer }}$ is equal to or less than $3.0 \times 10^{-7} \mathrm{erg} / \mathrm{cm}$. This is equivalent to spin-flop rotation for the aforementioned hysteresis. When $A_{\text {interlayer }}$ is between $4.0 \times 10^{-7} \mathrm{erg} / \mathrm{cm}$ and $8.0 \times 10^{-7} \mathrm{erg} / \mathrm{cm}$, the magnetization in the hard layer is reversed later than that of the soft layer, so this is equivalent to incoherent rotation. When $A_{\text {interlayer }}$ is stronger than $8.0 \times 10^{-7} \mathrm{erg} / \mathrm{cm}$, the D is almost 0 . This is equivalent to coherent rotation.

Finally, we discuss the magnetization reversal process as shown in Fig. 5, which corresponds to the incoherent rotation mode. Fig. 10 shows the schematic diagram of magnetization reversal process when $A_{\text {interlayer }}$ is $5.0 \times 10^{-7} \mathrm{erg} / \mathrm{cm}$. The recording layer is uniformly 


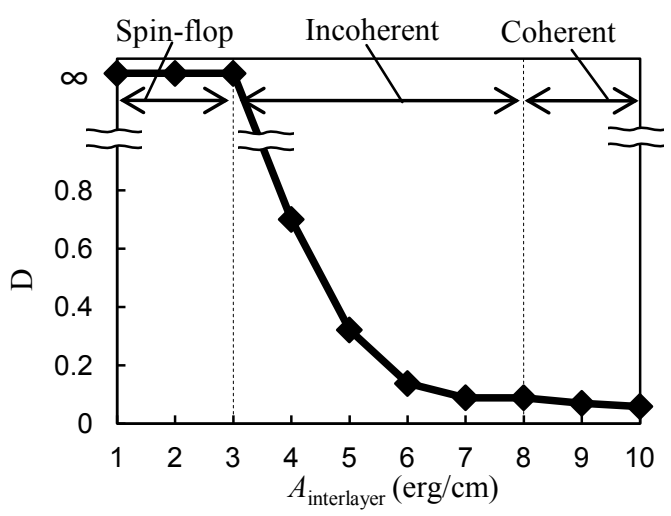

Fig. 9. Dependence of delay parameter D on $A_{\text {interlayer }}$.

magnetized by applying magnetic field for initial magnetization as shown in Fig. 10(a). After that, the patterned magnetic film of the master is in contact with the recording layer, and $H_{\mathrm{a}}$ is applied as shown in Fig. 10(b), which is equivalent to Fig. 5(b). Magnetization distribution of the soft layer does not coincide with the hard layer then. Effective magnetic field is expressed by

$$
\boldsymbol{H}_{\mathrm{eff}}^{\mathrm{h}(\mathrm{s})}=\boldsymbol{H}_{\mathrm{k}}^{\mathrm{h}(\mathrm{s})}+\boldsymbol{H}_{\mathrm{ex}}^{\mathrm{h}(\mathrm{s})}+\boldsymbol{H}_{\mathrm{d}}^{\mathrm{h}(\mathrm{s})}+\boldsymbol{H}_{\mathrm{r}}^{\mathrm{h}(\mathrm{s})},
$$

where $\boldsymbol{H}_{\mathrm{eff}}^{\mathrm{h}}$ is the effective field of the hard layer, $\boldsymbol{H}_{\mathrm{eff}}^{\mathrm{s}}$ isthe effective field of the soft layer, $\boldsymbol{H}_{\mathrm{k}}^{\mathrm{h}(\mathrm{s})}$ is the anisotropic field in each layer, $\boldsymbol{H}_{\mathrm{ex}}^{\mathrm{h}(\mathrm{s})}$ is the exchange field in each layer, $\boldsymbol{H}_{\mathrm{d}}^{\mathrm{h}(\mathrm{s})}$ is the magnetostatic field in each layer, and $\boldsymbol{H}_{\mathrm{r}}^{\mathrm{h}(\mathrm{s})}$ is the recording field in each layer. When the magnitude of the sum of $\boldsymbol{H}_{\mathrm{ex}}^{\mathrm{h}}, \boldsymbol{H}_{\mathrm{d}}^{\mathrm{h}}$ and $\boldsymbol{H}_{\mathrm{r}}^{\mathrm{h}}$ is stronger than $\left|\boldsymbol{H}_{\mathrm{k}}^{\mathrm{h}}\right|$, the magnetization reversal in the hard layer occurs. At area $\mathrm{P}$ shown in Fig. 10(b), simulation result reveals that $\left|\boldsymbol{H}_{\mathrm{k}}^{\mathrm{h}}\right|$ is larger than $\left|\boldsymbol{H}_{\mathrm{ex}}^{\mathrm{h}}+\boldsymbol{H}_{\mathrm{d}}^{\mathrm{h}}+\boldsymbol{H}_{\mathrm{r}}^{\mathrm{h}}\right|$. Therefore, magnetization in the hard layer is not reversed at $\mathrm{P}$ as shown in Fig. 10(b). After removal of $H_{\mathrm{a}}$ magnetization of the soft layer is affected by that of the hard layer and coincides with it as shown in Fig. 10(c), which is equivalent to Fig. 5(c). At area Q shown in Fig. 10(c), simulation result reveals that $\left|\boldsymbol{H}_{\mathrm{k}}^{\mathrm{s}}\right|$ is smaller than $\left|\boldsymbol{H}_{\mathrm{ex}}^{\mathrm{s}}+\boldsymbol{H}_{\mathrm{d}}^{\mathrm{s}}+\boldsymbol{H}_{\mathrm{r}}^{\mathrm{s}}\right|$. Therefore, magnetization in the soft layer is reversed again at Q as shown in Fig. 10(c).

\section{Conclusion}

It was found that magnetization reversal process drastically changes by varying interlayer exchange coupling between the soft and hard layers in the ECC media. In the case of intermediate value of the interlayer exchange coupling, during the application of printing field, magnetization in the soft layer initially reverses and magnetization in the hard layer follows that of the soft layer. Furthermore, after removal of printing field, magnetization in the soft layer is reversed again according with that in the hard layer.

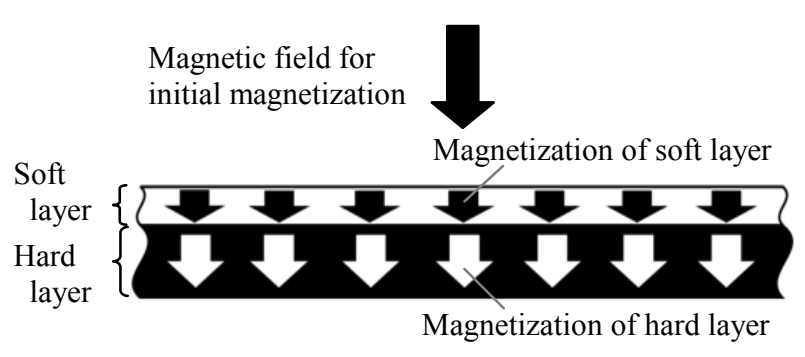

(a) Initial magnetization

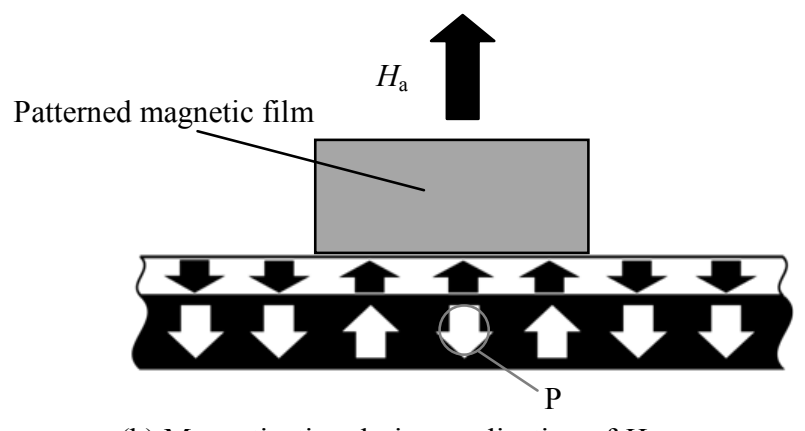

(b) Magnetization during application of $H_{\mathrm{a}}$

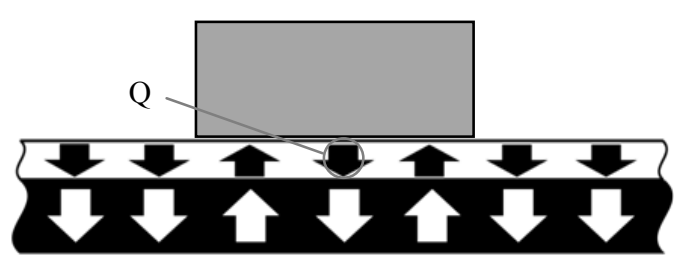

(c) Magnetization after removal of $H_{\mathrm{a}}$

Fig. 10. Schematic diagram of magnetization reversal process when $A_{\text {interlayer }}$ is $5.0 \times 10^{-7} \mathrm{erg} / \mathrm{cm}$.

\section{Acknowledgment}

This work was supported in part by the Grant-in-Aid for Scientific Research (C) (No. 24560394) from the Japan Society for the Promotion of Science (JSPS) of Japan.

\section{References}

1. R. H. Victora, and X. Shen, IEEE Trans. Magn., 41, 2828 (2005).

2. Y. Shiroishi, K. Fukuda, I. Tagawa, H. Iwasaki, S. Takenoiri, H. Tanaka, H. Mutoh, and N. Yoshikawa, IEEE Trans. Magn., 45, 3816 (2009).

3. A. Berger, N. Supper, Y. Ikeda, B. Lengsfield, A. Moser, and E. E. Fullerton, Appl. Phys. Lett., 93, 122502 (2008).

4. Y. Inaba, T. Shimatsu, S. Watanabe, O. Kitakami, S. Okamoto, H. Muraoka, H. Aoi, and Y. Nakamura, J. Magn. Soc. Jpn., 31, 178 (2007).

5. N. Sheeda, M. Nakazawa, H. Konishi, T. Komine, and R. Sugita, IEEE Trans. Magn., 45, 3676 (2009).

6. T. Komine, T. Murata, Y. Sakaguchi, and R. Sugita, IEEE Trans. Magn., 44, 3416 (2008). 